\section{It takes two to tango}

\author{
Wer führt den Tanz zwischen Gesellschaft und technologischer \\ Entwicklung im Zeitalter der Digitalisierung? Und welche Rolle \\ spielt die Nachhaltigkeit dabei? \\ Von Felix Sühlmann-Faul
}

\begin{abstract}
„Aber freilich: es ist eine fremde Macht über die Technik gekommen. Die Technik mit ihren unbegrenzten Möglichkeiten war die Versuchung. Die Entscheidung aber gab die Wirtschaft und ihre Zwecksetzung. " [1]
\end{abstract}

W ir befinden uns - unbestreitbar im Zeitalter der Digitalisierung. Spezialthemen wie Blockchain, künstliche Intelligenz oder die Beeinflussung von Wahlen durch die Hintertür sozialer Medien sind Phänomene, die inzwischen zum kollektiven Alltag gehören. Und viele Menschen fühlen sich abstrakt überfordert oder fürchten konkret um ihre Arbeit, die gegebenenfalls bald von einer Maschine schneller erledigt werden könnte. Dass die Digitalisierung auch viele Prozesse zu beschleunigen vermag, die eine ohnehin schon ausgebeutete und bedrohte Umwelt noch näher an den Kollaps führen, entgeht jedoch einer Thematisierung in der Öffentlichkeit. Politik und Wirtschaft schweigen sich ebenfalls größtenteils über den Zusammenhang zwischen Digitalisierung und Nachhaltigkeitsfragestellungen aus. Vielmehr steht das Abfeiern der Digitalisierung auf dem Programm als Heilsbringerin, als Retterin von Wirtschaftsstandorten, die im Vorbeigehen automatisch auch alles nachhaltiger macht. Sie zu ignorieren, zöge nie konkret formulierte, aber auf jeden Fall verheerende Folgen nach sich.

Es drängen sich Vergleiche zu anderen Kapiteln des Aushandlungsprozesses zwischen Gesellschaft und technologischer Entwicklung auf - einem Tanz, den manchmal die Gesellschaft, manchmal ökonomische oder technokratische Partner führten. Euphorisch herbeigefeiert war es „La Fée Electricité“ zum Ende des 19. Jahrhunderts, die vom damals erstarkten Bürgertum einen ähnlichen Stellenwert wie heute die Digitalisierung zugeschrieben bekam. Als reine Naturkraft und Befreierin der Menschheit wurde sie gerahmt, ungeahnt, dass die Elektrifizierung später größtenteils über die umweltschädliche Nutzung fossiler Energieträger zustande kommen würde. Auch die Atomkraft wurde in den 1960er Jahren durch überhitzte Erwartungsdynamiken gar als „Energiekreislauf" bezeichnet. Das Automobil hat der Menschheit eine unvergleichbare Erweiterung individueller Horizonte ermöglicht, ist aber inzwischen die Geißel gestresster Innenstadtbewohner/innen und trägt deutlich zum anthropogenen $\mathrm{CO}_{2}$-Ausstoß bei. Heute feiern wir die Möglichkeiten, die uns unsere kleinen Hochleistungscomputer in der Hosentasche ermöglichen, denken aber nicht darüber nach, wo die vielen smarten Gerätschaften nach ihrem frühen Ableben landen und dass Datenzentren inzwischen zu $80 \%$ damit beschäftigt sind, Netflix, YouTube und andere Streaminginhalte zu liefern.

Diese Beispiele zeigen, dass der erwähnte ,Tanz' zwischen Sozio- und Technosphäre ein Aushandlungsprozess sein muss, der nicht im Rhythmus der Ökonomie getanzt werden darf. Nachhaltigkeit ist ein elitäres Thema und dazu gehört auch eine nachhaltige Digitalisierung.
Wer überhaupt weiß beziehungsweise möchte wissen, dass das schicke Smartphone - zur Vertragsverlängerung vermeintlich für nur einen Euro - aus Rohstoffen besteht, die unter anderem einen Bürgerkrieg in der Demokratischen Republik Kongo finanzieren?

Die neoliberale Einschätzung, dass Nachhaltigkeit in der Hand der Konsument/innen liegt, ist ähnlich naiv, wie sich darauf zu stützen, dass Technik ja neutral sei. Auswirkungen und Zielsetzung (siehe Eingangszitat) sind keineswegs neutral. Um das Thema Nachhaltigkeit auch in den vielen Köpfen und Unternehmen, in denen es keine Rolle spielt, zu etablieren, braucht es politische Maßnahmen auf Basis wissenschaftlicher Faktenlage. Diese Maßnahmen sind kurzfristig vielleicht schmerzhaft, sichern aber längerfristig das Überleben der Menschheit. Denn es geht um nicht weniger.

Der Wissenschaftliche Beirat der Bundesregierung hat seinen aktuellen Bericht „Unsere digitale Zukunft“ am 12. 04. 2019 übergeben. Wie stets ist der Bericht exzellent geschrieben und stellt wohl eine der besten Quellen dar, sich mit dem Thema Digitalisierung und Nachhaltigkeit zu befassen. Für eine nachhaltige Digitalisierung muss die Steuerung der technologischen Entwicklung rein durch wirtschaftliche Interessen vermieden werden. Hoffen wir, dass dieser Bericht endlich eine nachhaltige Digitalisierung auf die politische Agenda rückt. Denn da gehört sie hin.

\section{Anmerkung}

[1] Clayton, John P. (Hrsg.) (1987): Paul Tillich: Main Works/Hauptwerke: Hauptwerke, in 6 Bdn., Bd.4, Religionsphilosophische Schriften, S. 186.

\section{AUTOR + KONTAKT}

Felix Sühlmann-Faul ist Techniksoziologe, Speaker und Autor mit der Spezialisierung auf Digitalisierung und Nachhaltigkeit.

Felix Sühlmann-Faul, Grünewaldstraße 2 a, 38104 Braunschweig. Tel.: +49 53122435134 , Website: www.suehlmann-faul.com copying and redistributing the material in any medium or format, provided the original work is properly cited, it is not used for commercial purposes and it is not remixed, transformed or built upon. The access to the digital version of this article is reserved to subscribers of ÖkologischesWirtschaften until two years after the date of publication; after two years it is available to all readers. 